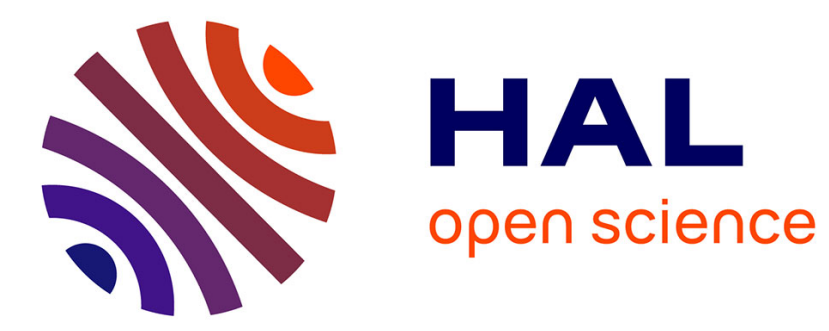

\title{
Bearing-Only Formation Control Using an SE(2) Rigidity Theory
}

Daniel Zelazo, Paolo Robuffo Giordano, Antonio Franchi

\section{To cite this version:}

Daniel Zelazo, Paolo Robuffo Giordano, Antonio Franchi. Bearing-Only Formation Control Using an SE(2) Rigidity Theory. IEEE Conf. on Decision and Control, CDC 2015 , Dec 2015, Osaka, Japan. hal-01187978

\section{HAL Id: hal-01187978 \\ https://inria.hal.science/hal-01187978}

Submitted on 28 Aug 2015

HAL is a multi-disciplinary open access archive for the deposit and dissemination of scientific research documents, whether they are published or not. The documents may come from teaching and research institutions in France or abroad, or from public or private research centers.
L'archive ouverte pluridisciplinaire HAL, est destinée au dépôt et à la diffusion de documents scientifiques de niveau recherche, publiés ou non, émanant des établissements d'enseignement et de recherche français ou étrangers, des laboratoires publics ou privés. 


\title{
Bearing-Only Formation Control Using an $S E(2)$ Rigidity Theory
}

\author{
Daniel Zelazo, Paolo Robuffo Giordano, Antonio Franchi
}

\begin{abstract}
This paper considers a formation control problem for a team of agents that are only able to sense the relative bearings from their local body frame to neighboring agents. It is further assumed that the sensing graph is inherently directed and a common reference frame is not known to all of the agents. Each agent is tasked with maintaining predetermined bearings with their neighbors. Using the recently developed rigidity theory for $S E(2)$ frameworks [1], we propose a gradient-type controller to stabilize the formation. The central construct in the $S E(2)$ rigidity theory for this work is the directed bearing rigidity matrix. We show that a necessary condition for the local stabilization of desired formation is for the corresponding $S E(2)$ framework to be minimally infinitesimally rigid.
\end{abstract}

\section{INTRODUCTION}

Formation control is one of the canonical problems studied in multi-agent coordination. At its most fundamental level, formation control involves the coordination of a team of agents to achieve some spatial formation shape. From a control systems perspective, the challenge in formation control is to find distributed strategies for the control and estimation of multi-agent systems that achieve a desired formation with guarantees on certain properties such as stability and performance.

Often the measurements available for each agent to achieve the task of formation control are non-linear functions of the state and inherently local in nature. Examples include range sensors for measuring the distance between agents, and bearing sensors for measuring the bearing angle from the body frame of one agent to another agent. Recently, there has been a growing interest in formation control problems using relative bearing sensing. Similar to problems in distance-constrained formation control [2]-[7], bearingconstrained formations employ a bearing rigidity theory (also referred to as parallel rigidity) for analysis. Whereas rigidity theory is useful for maintaining formations specified by fixed inter-agent distances, bearing rigidity attempts to keep the bearing vector between neighboring agents constant (with no constraints on the scale of the formation). Bearing rigidity was used in [8]-[11] for deriving distributed control laws for controlling formations with bearing measurements. Bearing rigidity has also proven useful for stabilization of formations using direction-only or line-of-sight only constraints [12][15]. Extensions of the bearing rigidity theory to arbitrary

Daniel Zelazo is with the Faculty of Aerospace Engineering at the Technion-Israel Institute of Technology, Haifa, Israel dzelazodtechnion.ac.il. Paolo Robuffo Giordano is with CNRS at Irisa and Inria Rennes Bretagne Atlantique, Campus de Beaulieu, 35042 Rennes Cedex, France prgeirisa.fr. Antonio Franchi is with CNRS-LAAS, 7 avenue du colonel Roche, F-31400 Toulouse, France antonio.franchi@laas.fr. dimensional ambient spaces for formation control was considered in [16], [17].

In this work, we explore an extension of rigidity theory for frameworks that are embedded in the special Euclidean group $S E(2)=\mathbb{R}^{2} \times \mathcal{S}^{1}$. This extension explicitly handles frameworks where the underlying graph is directed and bearings are expressed in the local frame of each $S E(2)$ point in the framework. The $S E(2)$ rigidity theory was first considered in our previous work [1], and we provide here new results on the structure of the corresponding directed bearing rigidity matrix. The main focus of this work is to exploit the $S E(2)$ rigidity theory to derive a distributed formation control strategy that drives a team of agents to a desired bearing-constrained formation. The formation is specified in terms of desired bearing vectors expressed in the local frame of each agent, and agents have access to bearing measurements also expressed in their local frame. Furthermore, the control requires no use of range measurements, in contrast to the strategy proposed in [12]. An almost global stability analysis of the proposed control is given and we also provide numerous numerical simulations illustrating the more subtle points of the result.

Preliminaries and Notations: The set of real numbers will be denoted as $\mathbb{R}$, the 1-dimensional manifold on the unit circle as $\mathcal{S}^{1}$, and $S E(2)=\mathbb{R}^{2} \times \mathcal{S}^{1}$ is the Special Euclidean Group 2. The standard Euclidean 2-norm for vectors is denoted $\|$.$\| . The null-space of a matrix A$ is denoted Null $[A]$. The block diagonal concatenation of a finite set of matrices $\left\{A_{1}, \ldots, A_{r}\right\}$ is denoted $\operatorname{diag}\left(A_{i}\right)$. Directed graphs and the matrices associated with them will be widely used in this work [18]. A directed graph $\mathcal{G}$ is specified by a vertex set $\mathcal{V}$, and an edge set $\mathcal{E} \subseteq \mathcal{V} \times \mathcal{V}$. The neighborhood of the vertex $i$ is the set $\mathcal{N}_{i}=\{j \in \mathcal{V} \mid(i, j) \in \mathcal{E}\}$, and the out-degree of vertex $i$ is $d_{\text {out }}(i)=\left|\mathcal{N}_{i}\right|$. The incidence matrix $E(\mathcal{G}) \in \mathbb{R}^{|\mathcal{V}| \times|\mathcal{E}|}$ is a $\{0, \pm 1\}$-matrix with rows and columns indexed by the vertices and edges of $\mathcal{G}$ [18]. Similarly, we define $E_{\text {out }}(\mathcal{G}) \in \mathbb{R}^{|\mathcal{V}| \times|\mathcal{E}|}$ to be a $\{0,+1\}$ matrix such that $\left[E_{\text {out }}(\mathcal{G})\right]_{i j}=1$ if node $i$ is the head of edge $j$, and 0 otherwise. We also introduce the notation $\bar{E}=E(\mathcal{G}) \otimes I_{2}$ and $\bar{E}_{\text {out }} \otimes I_{2}$. The complete directed graph, denoted $K_{|\mathcal{V}|}$ is a graph with all possible directed edges (i.e. $|\mathcal{E}|=|\mathcal{V}|(|\mathcal{V}|-1))$

\section{RIgIDITY THEORY FOR $S E(2)$ FRAMEWORKS}

Rigidity is a combinatorial theory for characterizing the stiffness or flexibility of structures formed by rigid bodies connected by flexible linkages or hinges [19]. Recently, there has been a growing interest in formation control strategies using bearings leading to the development of the bearing 
rigidity theory [8], [11], [15], [16]. In this work we employ an extension of these works to frameworks embedded in the Special Euclidean Group $S E(2)$, originally considered in our previous work [1]. To begin, we first formally define frameworks for points in $S E(2)$.

Definition II.1. An $S E(2)$ framework is the triple $(\mathcal{G}, p, \psi)$, where $\mathcal{G}=(\mathcal{V}, \mathcal{E})$ is a directed graph, $p: \mathcal{V} \rightarrow \mathbb{R}^{2}$ and $\psi:$ $\mathcal{V} \rightarrow \mathcal{S}^{1}$ maps each vertex to a point in $S E(2)=\mathbb{R}^{2} \times \mathcal{S}^{1}$.

We denote by $\chi_{v}=\left(p_{v}, \psi_{v}\right) \in S E(2)$ the position and attitude vector of node $v \in \mathcal{V}$. For notational convenience, we will refer to the vectors $\chi_{p}=p(\mathcal{V}) \in \mathbb{R}^{2|\mathcal{V}|}$ and $\chi_{\psi}=$ $\psi(\mathcal{V}) \in \mathcal{S}^{\mid \mathcal{| V |}}$ as the position and attitude components of the complete framework configuration. The vector $\chi(\mathcal{V}) \in$ $S E(2)^{|\mathcal{V}|}$ is the stacked position and attitude vector for the complete framework. We also denote by $\chi_{p}^{x} \in \mathbb{R}^{|\mathcal{V}|}\left(\chi_{p}^{y}\right)$ as the $x$-coordinate ( $y$-coordinate) vector for the framework configuration.

In the literature on bearing rigidity, the bearings between points in the framework are often expressed in a global frame. For $S E(2)$ rigidity, formations will be specified in terms of relative bearing vectors between points in the framework with respect to the local frame of each point. ${ }^{1}$ This is motivated by application scenarios where the sensing is inherently expressed in the local frame of each agent and also justifies the explicit use of directed graphs in the definition.

In this venue, we assume that a point $\chi_{v} \in S E(2)$ has a bearing measurement of the point $\chi_{u}$ if and only if the directed edge $(v, u)$ belongs to the graph $\mathcal{G}$ (i.e., $(v, u) \in$ $\mathcal{E})$; this measurement is denoted by $r_{v u} \in \mathcal{S}^{2}$. The relative bearing is measured from the body coordinate system of that point.

We define the directed bearing rigidity function associated with the $S E(2)$ framework, $b_{\mathcal{G}}: S E(2)^{|\mathcal{V}|} \rightarrow \mathcal{S}^{2|\mathcal{E}|}$, as

$$
b_{\mathcal{G}}(\chi(\mathcal{V}))=\left[\begin{array}{lll}
r_{e_{1}}^{T} & \cdots & r_{e_{|\mathcal{E}|}^{T}}^{T}
\end{array}\right]^{T}
$$

we use the notation $e_{i} \in \mathcal{E}$ to represent a directed edge in the graph and assume a labeling of the edges in $\mathcal{G}$.

Observe that the bearing measurement can be expressed directly in terms of the relative positions and attitudes of the points expressed in the world frame,

$$
\begin{aligned}
r_{v u} & =\left[\begin{array}{cc}
\cos \left(\psi_{v}\right) & \sin \left(\psi_{v}\right) \\
-\sin \left(\psi_{v}\right) & \cos \left(\psi_{v}\right)
\end{array}\right] \frac{\left(p_{u}-p_{v}\right)}{\left\|p_{v}-p_{u}\right\|} \\
& =T\left(\psi_{v}\right)^{T} \frac{\left(p_{u}-p_{v}\right)}{\left\|p_{v}-p_{u}\right\|}=T\left(\psi_{v}\right)^{T} \bar{p}_{v u},
\end{aligned}
$$

where the matrix $T\left(\psi_{v}\right)$ is a rotation matrix from the world frame to the body frame of agent $v$, and $\bar{p}_{v u}$ is a shorthand notation for the unit-length relative position vector from $v$ to $u$. Furthermore, the directed bearing rigidity function can also be expressed as

$$
b_{\mathcal{G}}(\chi(\mathcal{V}))=\operatorname{diag}\left(\frac{T\left(\psi_{v}\right)^{T}}{\left\|p_{v}-p_{u}\right\|}\right) \bar{E}^{T} \chi_{p} .
$$

\footnotetext{
${ }^{1}$ In our previous work [1], bearings were expressed as angles rather than vectors.
}

We now review formal definitions for $S E(2)$ rigidity [1].

Definition II.2 (Rigidity in $S E(2))$. The $S E(2)$ framework $(\mathcal{G}, p, \psi)$ is rigid in $S E(2)$ if there exists a neighborhood $S$ of $\chi(\mathcal{V}) \in S E(2)^{|\mathcal{V}|}$ such that

$$
b_{K_{|\mathcal{V}|}}^{-1}\left(b_{K_{|\mathcal{V}|}}(\chi(\mathcal{V}))\right) \cap S=b_{\mathcal{G}}^{-1}\left(b_{\mathcal{G}}(\chi(\mathcal{V}))\right) \cap S,
$$

where $b_{K_{|\mathcal{V}|}}^{-1}\left(b_{K_{|\mathcal{V}|}}(\chi(\mathcal{V}))\right) \subset S E(2)$ denotes the pre-image of the point $b_{K_{|\mathcal{V}|}}(\chi(\mathcal{V}))$ under the directed bearing rigidity map.

The $S E(2)$ framework $(\mathcal{G}, p, \psi)$ is roto-flexible in $S E(2)$ if there exists an analytic path $\eta:[0,1] \rightarrow S E(2)^{|\mathcal{V}|}$ such that $\eta(0)=\chi(\mathcal{V})$ and

$$
\eta(t) \in b_{\mathcal{G}}^{-1}\left(b_{\mathcal{G}}(\chi(\mathcal{V}))\right)-b_{K_{|\mathcal{V}|}}^{-1}\left(b_{K_{|\mathcal{V}|}}(\chi(\mathcal{V}))\right) \forall t \in(0,1] .
$$

This definition states that an $S E(2)$ framework $(\mathcal{G}, p, \psi)$ is rigid if and only if for any point $q \in S E(2)$ sufficiently close to $\chi(\mathcal{V})$ with $b_{\mathcal{G}}(\chi(\mathcal{V}))=b_{\mathcal{G}}(q)$, that there exists a local bearing preserving map of $S E(2)$ taking $\chi(\mathcal{V})$ to $q$. The term roto-flexible is used to emphasize that an analytic path in $S E(2)$ can consist of motions in the plane in addition to angular rotations about the body axis of each point.

Definition II.3 (Equivalent and Congruent $S E(2)$ Frameworks). Frameworks $(\mathcal{G}, p, \psi)$ and $(\mathcal{G}, q, \phi)$ are bearing equivalent if

$$
T\left(\psi_{u}\right)^{T} \bar{p}_{u v}=T\left(\phi_{u}\right)^{T} \bar{q}_{u v},
$$

for all $(u, v) \in \mathcal{E}$ and are bearing congruent if

$T\left(\psi_{u}\right)^{T} \bar{p}_{u v}=T\left(\phi_{u}\right)^{T} \bar{q}_{u v}$ and $T\left(\psi_{v}\right)^{T} \bar{p}_{v u}=T\left(\phi_{v}\right)^{T} \bar{q}_{v u}$,

for all $(u, v) \in \mathcal{V}$ with $u \neq v$.

Definition II.4 (Global rigidity of $S E(2)$ Frameworks). A framework $(\mathcal{G}, p, \psi)$ is globally rigid in $S E(2)$ if every framework which is bearing equivalent to $(\mathcal{G}, p, \psi)$ is also bearing congruent to $(\mathcal{G}, p, \psi)$.

The notion of infinitesimal rigidity is characterized by the null-space of the Jacobian of the directed bearing rigidity function, $\nabla_{\chi} b_{\mathcal{G}}(\chi(\mathcal{V}))$. In this direction, define the directed bearing rigidity matrix, $\mathcal{B}_{\mathcal{G}}(\chi(\mathcal{V}))$, as

$$
\mathcal{B}_{\mathcal{G}}(\chi(\mathcal{V})):=\nabla_{\chi} b_{\mathcal{G}}(\chi(\mathcal{V})) \in \mathbb{R}^{2|\mathcal{E}| \times 3|\mathcal{V}|}
$$

It is worth examining the structure of this matrix in more detail. In particular, we have that

$$
\begin{aligned}
& \frac{\partial r_{v u}}{\partial \chi_{v}}=\left[\begin{array}{ll}
-\frac{r_{v u}^{\perp}\left(r_{v u}^{\perp}\right)^{T}}{\left\|p_{u}-p_{v}\right\|} T\left(\psi_{v}\right)^{T} & -r_{v u}^{\perp}
\end{array}\right] \\
& \frac{\partial r_{v u}}{\partial \chi_{u}}=\left[\begin{array}{ll}
\frac{r_{v u}^{\perp}\left(r_{v u}^{\perp}\right)^{T}}{\left\|p_{u}-p_{v}\right\|} T\left(\psi_{v}\right)^{T} & \mathbf{0}
\end{array}\right]
\end{aligned}
$$

Here, $r_{v u}^{\perp}=T(\pi / 2) r_{v u}$ denotes a $\pi / 2$ counterclockwise rotation of the vector $r_{v u}$. Furthermore, the matrix $r_{v u}^{\perp}\left(r_{v u}^{\perp}\right)^{T}$ is a projection matrix, and we introduce the notation $P_{r_{v u}}=$ $I_{2}-r_{v u} r_{v u}^{T}=r_{v u}^{\perp}\left(r_{v u}^{\perp}\right)^{T}$. For notational convenience, we will also often work with a permutation of the directed bearing rigidity matrix,

$$
\begin{gathered}
\tilde{\mathcal{B}}_{\mathcal{G}}(\chi(\mathcal{V}))=\left[\begin{array}{ll}
\nabla_{\chi_{p}} b_{\mathcal{G}}(\chi(\mathcal{V})) & \nabla_{\chi_{\psi}} b_{\mathcal{G}}(\chi(\mathcal{V}))
\end{array}\right] \\
=\left[\begin{array}{ll}
-\operatorname{diag}\left(\frac{P_{r_{v u}}}{\left\|p_{u}-p_{v}\right\|} T\left(\psi_{v}\right)^{T}\right) \bar{E}^{T}-\operatorname{diag}\left(r_{\text {vu }}^{\perp}\right) E_{\text {out }}^{T}
\end{array}\right] .
\end{gathered}
$$


Definition II.5 (Infinitesimal Rigidity in $S E(2))$. An $S E(2)$ framework $(\mathcal{G}, p, \psi)$ is infinitesimally rigid if $\operatorname{Null}\left[\mathcal{B}_{\mathcal{G}}(\chi(\mathcal{V}))\right]=\operatorname{Null}\left[\mathcal{B}_{K_{|\mathcal{V}|}}(\chi(\mathcal{V}))\right]$. Otherwise, it is infinitesimally roto-flexible in $S E(2)$.

Definition II.5 leads to the following result which relates the infinitesimal rigidity of an $S E(2)$ framework to the rank of the directed bearing rigidity matrix.

Theorem II.6 ( [1]). An $S E(2)$ framework is infinitesimally rigid if and only if $\mathbf{r k}\left[\mathcal{B}_{\mathcal{G}}(\chi(\mathcal{V}))\right]=3|\mathcal{V}|-4$.

For an infinitesimally rigid $S E(2)$ framework, the null space of the directed bearing rigidity matrix is also well understood. It corresponds to the rigid body translations and dilations of the framework, in addition to coordinated rotations. The translations and dilations correspond precisely to the infinitesimal motions required in bearing rigidity for frameworks embedded in $\mathbb{R}^{2}$ [13]. A coordinated rotation consists of a rotation of each agent about its own body axis at the same angular speed coupled with a rigid-body rotation of the framework in $\mathbb{R}^{2}$ so as to leave unchanged all the relative bearings. These coordinated rotations are the nontrivial solutions to the equation

$-\operatorname{diag}\left(\frac{P_{r_{v u}}}{\left\|p_{u}-p_{v}\right\|} T\left(\psi_{v}\right)^{T}\right) \bar{E}^{T} p=\operatorname{diag}\left(r_{v u}^{\perp}\right) E_{\text {out }}^{T} \psi$.

The solutions define the coordinated rotation subspace,

$$
\mathcal{R}_{\circlearrowright}=\left\{\left[\begin{array}{l}
p \\
\psi
\end{array}\right] \mid\left[\begin{array}{l}
p \\
\psi
\end{array}\right] \text { is a solution of }(6)\right\} \text {. }
$$

It was shown in [1] that $\operatorname{dim}\left\{\mathcal{R}_{\circlearrowright}\right\}=1$ if and only if the $S E(2)$ framework is infinitesimally rigid.

Proposition II.7. For an infinitesimally rigid SE(2) framework,

$$
\operatorname{Null}\left[\tilde{\mathcal{B}}_{\mathcal{G}}(\chi(\mathcal{V}))\right]=\operatorname{span}\left\{\left[\begin{array}{c}
\boldsymbol{1}_{\mathcal{V}} \otimes I_{2} \\
\mathbf{0}
\end{array}\right],\left[\begin{array}{c}
\chi_{p} \\
\mathbf{0}
\end{array}\right], \mathcal{R}_{\circlearrowright}\right\} .
$$

We are also able to define the notion of minimally infinitesimal rigid $S E(2)$ frameworks. In fact, this definition follows from Theorem II.6 since an infinitesimally rigid $S E(2)$ framework will require at least $|\mathcal{E}|=3|\mathcal{V}|-4$ edges.

Definition II.8 (Minimal Infinitesimal Rigidity in $S E(2)$ ). An $S E(2)$ framework $(\mathcal{G}, p, \psi)$ is minimally infinitesimally rigid $(M I R)$ if for any $\mathcal{G}^{\prime}$ obtained by removing any edge from $\mathcal{G}$, the resulting framework $\left(\mathcal{G}^{\prime}, p, \psi\right)$ is infinitesimally rotoflexible in $S E(2)$.

Finally, observe that the directed bearing rigidity matrix is defined in terms of the bearing vectors expressed in the local frame of each agent $\left(r_{v u}\right)$, and the distance between agents. We also define the scale-free directed bearing rigidity matrix. This is motivated by the desire to implement control strategies that do not depend on range, as we will see in the sequel. The scale-free bearing rigidity matrix is defined as

$$
\hat{\mathcal{B}}_{\mathcal{G}}(\chi(\mathcal{V}))=\left[-\operatorname{diag}\left(P_{r_{v u}} T\left(\psi_{v}\right)^{T}\right) \bar{E}^{T}-\operatorname{diag}\left(r_{v u}^{\perp}\right) E_{\text {out }}^{T}\right] .
$$

Proposition II.9. For a $S E(2)$ framework $(\mathcal{G}, p, \psi)$ with $p_{u} \neq p_{v}$ for all $u, v \in \mathcal{V}$, one has

$$
\mathbf{r k}\left[\mathcal{B}_{\mathcal{G}}(\chi(\mathcal{V}))\right]=\mathbf{r k}\left[\hat{\mathcal{B}}_{\mathcal{G}}(\chi(\mathcal{V}))\right]
$$

Proof. The result follows directly from the relationship

$$
\hat{\mathcal{B}}_{\mathcal{G}}(\chi(\mathcal{V}))=\left[\begin{array}{cc}
\operatorname{diag}\left(\left\|p_{u}-p_{v}\right\|\right) & \mathbf{0} \\
\mathbf{0} & I_{|\mathcal{V}|}
\end{array}\right] \tilde{\mathcal{B}}_{\mathcal{G}}(\chi(\mathcal{V})) .
$$

\section{An Illustrative Example: The Triangle}

To illustrate some of the above definitions, we consider an $S E(2)$ framework consisting of three nodes, i.e., a triangle.

Proposition II.10. An $S E(2)$ framework $(\mathcal{G}, p, \psi)$ consisting of three non-collinear points is infinitesimally rigid if and only if $|\mathcal{E}|=5$.

Proof. Assume, without loss of generality, that $\mathcal{E}=$ $\left\{\left(v_{1}, v_{2}\right),\left(v_{1}, v_{3}\right),\left(v_{2}, v_{1}\right),\left(v_{2}, v_{3}\right),\left(v_{3}, v_{1}\right)\right\}$, i.e., the edge $\left(v_{2}, v_{3}\right)$ is "missing" from $\mathcal{E}$. We now show that the bearing $r_{\left(v_{2}, v_{3}\right)}$ can be algebraically determined from the other five bearing measurements. Define the angle $\beta_{u v}=$ $\operatorname{atan} 2\left(r_{u v}^{x}, r_{u v}^{y}\right)$, i.e., $\left.r_{u v}=\left[\cos \left(\beta_{u v}\right) \sin \left(\beta_{u v}\right)\right)\right]^{T}$. It is easy to check that the following holds:

$$
\beta_{u v}-\beta_{v u}=\psi_{v}-\psi_{u}-\pi \text { for any } u, v .
$$

Exploiting (8) three times yields

$$
\begin{aligned}
\beta_{v_{2} v_{3}} & =\beta_{v_{3} v_{2}}+\psi_{v_{3}}-\psi_{u_{2}}-\pi \\
& =\beta_{v_{3} v_{2}}+\left(\psi_{v_{3}}-\psi_{v_{1}}\right)-\left(\psi_{u_{2}}-\psi_{v_{1}}\right)-\pi \\
& =\beta_{v_{3} v_{2}}+\beta_{v_{1} v_{3}}-\beta_{v_{3} v_{1}}-\beta_{v_{1} v_{2}}+\beta_{v_{2} v_{1}}-\pi,
\end{aligned}
$$

which proves that $\beta_{v_{2} v_{3}}$, and therefore $r_{v_{2} v_{3}}$, can be computed from the five available bearings. Therefore, measuring five bearings is equivalent to measuring six bearings, i.e., to having a complete measurement graph. To conclude the first part of the proof we observe that if the agents are not aligned then the complete graph guarantees the infinitesimal rigidity of the framework.

In order to show minimality, we first observe that each two rows of the bearing rigidity matrix corresponding to each measured bearing $r_{u v}$ are linearly dependent. In fact, this can be seen by noticing that $\left(r_{u v}\right)^{T}$ is in the left nullspace of the $2 \times 6$ matrix composed by these two rows. Furthermore, as stated by Theorem II.6, the rank of the bearing rigidity matrix must be in this case $3 \cdot 3-4=5$ in order to have infinitesimal rigidity. Therefore, the presence of at least five bearing measurements is necessary in order to have infinitesimal rigidity. This proves the minimality of the framework and concludes the proof.

\section{Formation CONTROL IN $S E(2)$}

We now study a formation control problem in $S E(2)$. Consider a team of $n$ agents $(n \geq 2)$ in $S E(2)$ where there is no knowledge of a common reference frame. The dynamics of each agent are expressed as

$$
\left[\begin{array}{c}
T\left(\psi_{i}\right)^{T} \dot{p}_{i} \\
\dot{\psi}_{i}
\end{array}\right]=\left[\begin{array}{c}
u_{i} \\
\omega_{i}
\end{array}\right], i=1, \ldots, n .
$$


Here, the control input $u_{i}$ is applied in the body-frame of agent $i$, and $w_{i}$ directly controls the angular velocity of agent $i$. Agents are able to sense the bearing to neighboring agents according to a fixed directed graph $\mathcal{G}=(\mathcal{V}, \mathcal{E})$.

We would like to design a distributed control law that utilizes only bearing information to drive the formation to a configuration that is congruent to the desired configuration (i.e., admits the same directed bearing rigidity function). We denote the desired formation in terms of desired relative bearings between each agent,

$$
\mathbf{b}_{\mathcal{G}}^{d}=\left[\begin{array}{lll}
\left(r_{e_{1}}^{d}\right)^{T} & \cdots & \left(r_{e_{|\mathcal{E}|}}^{d}\right)^{T}
\end{array}\right]^{T} .
$$

Assumption 1. There exists an $S E(2)$ framework $\left(\mathcal{G}, p^{d}, \psi^{d}\right)$ with $\chi^{d}(\mathcal{V})=\left(p^{d}, \psi^{d}\right)$ such that $b_{\mathcal{G}}\left(\chi^{d}(\mathcal{V})\right)=\mathbf{b}_{\mathcal{G}}^{d}$. Furthermore, the directed bearing rigidity matrix $\tilde{\mathcal{B}}_{\mathcal{G}}\left(\chi^{d}(\mathcal{V})\right)$ is $M I R$ in $S E(2)$.

In this direction, define the following potential function,

$$
J(\chi(\mathcal{V}))=\frac{1}{2}\left\|b_{\mathcal{G}}(\chi(\mathcal{V}))-\mathbf{b}_{\mathcal{G}}^{d}\right\|^{2} .
$$

We would like to examine the following gradient controller,

$$
\begin{aligned}
{\left[\begin{array}{c}
\dot{p} \\
\dot{\psi}
\end{array}\right] } & =-k \nabla_{\chi} J(\chi(\mathcal{V}))=-k \tilde{\mathcal{B}}_{\mathcal{G}}(\chi(\mathcal{V}))^{T}\left(b_{\mathcal{G}}(\chi)-\mathbf{b}_{\mathcal{G}}^{d}\right) \\
& =-k \tilde{\mathcal{B}}_{\mathcal{G}}(\chi(\mathcal{V}))^{T}\left(b_{\mathcal{G}}(\chi)-\mathbf{b}_{\mathcal{G}}^{d}\right),
\end{aligned}
$$

Here, $k>0$ is a scalar gain used to improve the rate of convergence of the system. For analysis purposes, we take $k=1$. Observe that by construction $\tilde{\mathcal{B}}_{\mathcal{G}}(\chi(\mathcal{V}))^{T} b_{\mathcal{G}}(\chi)=0$, leading to

$$
\left[\begin{array}{c}
\dot{p} \\
\dot{\psi}
\end{array}\right]=\tilde{\mathcal{B}}_{\mathcal{G}}(\chi(\mathcal{V}))^{T} \mathbf{b}_{\mathcal{G}}^{d}
$$

Note that this control is expressed in the global frame. Expressed in the local body frame, the controller takes the expression

$$
\begin{aligned}
& u_{i}=T\left(\psi_{i}\right)^{T} \dot{p}_{i}=-T\left(\psi_{i}\right)^{T} \sum_{(i, j) \in \mathcal{E}} T\left(\psi_{i}\right) \frac{P_{r_{i j}}}{\left\|p_{j}-p_{i}\right\|} r_{i j}^{d} \\
& +T\left(\psi_{i}\right)^{T} \sum_{(j, i) \in \mathcal{E}} T\left(\psi_{j}\right) \frac{P_{r_{j i}}}{\left\|p_{i}-p_{j}\right\|} r_{j i}^{d} \\
& =-\sum_{(i, j) \in \mathcal{E}} \frac{P_{r_{i j}}}{\left\|p_{j}-p_{i}\right\|} r_{i j}^{d}+\sum_{(j, i) \in \mathcal{E}} T\left(\psi_{j}-\psi_{i}\right) \frac{P_{r_{j i}}}{\left\|p_{i}-p_{j}\right\|} r_{j i}^{d} \\
& \dot{\psi}_{i}=-\sum_{(i, j) \in \mathcal{E}}\left(r_{i j}^{\perp}\right)^{T} r_{i j}^{d}
\end{aligned}
$$

A few comments regarding the above control strategy are in order. Indeed, the control in (12), (13) has a distributed structure depending only on the sensing graph $\mathcal{G}$. On the other hand, this control requires communication between agents. That is, if there is an edge $(j, i) \in \mathcal{E}$, then agent $i$ requires the bearing measurement $r_{j i}$ and the desired bearing $r_{j i}^{d}$ from agent $j$. Furthermore, the agents also require information on their relative orientation, $T\left(\psi_{i}\right)^{T} T\left(\psi_{j}\right)=$
$T\left(\psi_{j}-\psi_{i}\right)$, as well as the distance $\left\|p_{i}-p_{j}\right\|$ between neighboring agents. As well-known, this latter quantity cannot be recovered from sole measured bearings and an independent measurement (via, e.g., a distance sensor) would be required. To cope with this issue, we will detail in the following a scale-free version of controller (12) for which no distance measurement is needed. On the other hand, the relative orientation $T\left(\psi_{j}-\psi_{i}\right)$ among neighboring pairs can be directly obtained in terms of measured bearings thanks to the rigidity of the framework $(\mathcal{G}, p, \psi)$. If $(\mathcal{G}, p, \psi)$ is rigid then one could, for instance, exploit the distributed estimation strategy illustrated in [1] for recovering the quantity $T\left(\psi_{j}-\psi_{i}\right)$ from the measured bearings. Alternatively, one could make use of the geometric arguments of [20] for algebraically obtaining $T\left(\psi_{j}-\psi_{i}\right)$ from the available bearings. An example of this algebraic procedure for the case of 3 agents is given in the proof of Proposition II.10.

In this direction, we now propose the following scale-free alternative control for avoiding measurement of the interagent distances as in (12),

$$
\begin{aligned}
T\left(\psi_{i}\right)^{T} \dot{p}_{i} & =-\sum_{(i, j) \in \mathcal{E}} P_{r_{i j}} r_{i j}^{d}+\sum_{(j, i) \in \mathcal{E}} T\left(\psi_{i}-\psi_{j}\right)^{T} P_{r_{j i}} r_{j i}^{d} \\
\dot{\psi}_{i} & =-\sum_{(i, j) \in \mathcal{E}}\left(r_{i j}^{\perp}\right)^{T} r_{i j}^{d}
\end{aligned}
$$

and we assume for the purpose of analysis that the agents are able to acquire their relative orientation This control can be expressed in a compact notation using the scale-free bearing rigidity matrix as

$$
\left[\begin{array}{c}
\operatorname{diag}\left(T\left(\psi_{i}\right)^{T}\right) \dot{p} \\
\dot{\psi}
\end{array}\right]=\left[\begin{array}{cc}
\operatorname{diag}\left(T\left(\psi_{i}\right)^{T}\right) & \mathbf{0} \\
\mathbf{0} & I_{|\mathcal{V}|}
\end{array}\right] \hat{\mathcal{B}}_{\mathcal{G}}(\chi)^{T} \mathbf{b}_{\mathcal{G}}^{d}
$$

It is worth noting that this control is in fact different than the one proposed in [16]. In particular, in [16] a consensustype algorithm is used to align all agents to a common orientation, thereby creating an effective common reference frame, while the control action in (13) does not enforce any agreement/alignment over a common orientation.

Before proceeding with a stability analysis of this control, we first present a useful result relating to the centroid and scale of the formation.

Proposition III.1. [16] The centroid of the formation $\bar{p}=$ $\frac{1}{n}\left(\boldsymbol{I}^{T} \otimes I_{2}\right) p$ and its scale $s_{p}=\frac{1}{n} \sqrt{\sum_{i=1}^{n}\left\|p_{i}-\bar{p}\right\|^{2}}$ are invariant under the dynamics (16).

Proof. In the global coordinate frame, the centroid dynamics can be expressed as

$\dot{\bar{p}}=\frac{\left(\mathbf{1}^{T} \otimes I_{2}\right)}{n} \dot{p}=-\frac{\left(\mathbf{1}^{T} \otimes I_{2}\right) \bar{E} \operatorname{diag}\left(T\left(\psi_{v}\right) P_{r_{v u}}\right)}{n} \mathbf{b}_{\mathcal{G}}^{d}=0$.

Similarly, the scale dynamics can be expressed as

$$
\dot{s}=\frac{1}{n} \frac{(p-\mathbf{1} \otimes \bar{p})^{T}}{\|p-\mathbf{1} \otimes \bar{p}\|} \dot{p} .
$$

From Proposition II.7, it follows that $p^{T} \dot{p}=0$ and $(\mathbf{1} \otimes$ $\bar{p})^{T} \dot{p}=0$ concluding the proof. 
We are now prepared to state the main result. We will show that, for almost all initial conditions, the dynamics in (16) asymptotically converges to the desired configuration.

Theorem III.2. Consider an MIR SE(2) framework $(\mathcal{G}, p(0), \psi(0))$ with directed bearing rigidity function $b_{\mathcal{G}}(\chi(\mathcal{V}))$ and a formation in $S E(2)$ specified by the vector of relative bearings $\mathbf{b}_{\mathcal{G}}^{d}$ satisfying Assumption 1. Then for almost all initial conditions $(p(0), \psi(0))$, the system (16) asymptotically converges to a configuration $\chi^{*}$ with $b_{\mathcal{G}}\left(\chi^{*}\right)=\mathbf{b}_{\mathcal{G}}^{d}$.

Proof. Denote the bearing error vector as $\delta=b_{\mathcal{G}}(\chi(\mathcal{V}))-$ $\mathbf{b}_{\mathcal{G}}^{d}$. The error dynamics can be expressed as

$$
\dot{\delta}=-\tilde{\mathcal{B}}_{\mathcal{G}}(\chi(\mathcal{V})) \hat{\mathcal{B}}_{\mathcal{G}}(\chi(\mathcal{V}))^{T} \delta
$$

Consider the quadratic Lyapunov function $V=\frac{1}{2} \delta^{T} \delta$ and note that the level sets $\{\delta \mid V(\delta) \leq c<\infty\}$ are compact and positively invariant. Furthermore, from Assumption 1, we can assume there exists a point $\chi^{d}(\mathcal{V})=\left(p^{d}, \psi^{d}\right)$ satisfying $b_{\mathcal{G}}\left(\left(p^{d}, \psi^{d}\right)\right)=\mathbf{b}_{\mathcal{G}}^{d}$ that has the same scale and centroid as the initial condition of the system. In particular, the point $\chi(\mathcal{V})=\chi^{d}(\mathcal{V})$ corresponds to the equilibrium point $\delta=$ 0 of (17). Evaluating the Lie derivative of $V$ yields $\dot{V}=$ $-\delta^{T}\left(\hat{\mathcal{B}}_{\mathcal{G}}(\chi(\mathcal{V})) \tilde{\mathcal{B}}_{\mathcal{G}}(\chi(\mathcal{V}))^{T}+\tilde{\mathcal{B}}_{\mathcal{G}}(\chi(\mathcal{V})) \hat{\mathcal{B}}_{\mathcal{G}}(\chi(\mathcal{V}))^{T}\right) \delta \leq 0$. To show this inequality, let $Q=-\tilde{\mathcal{B}}_{\mathcal{G}}(\chi(\mathcal{V})) \tilde{\mathcal{B}}_{\mathcal{G}}(\chi(\mathcal{V}))^{T}$ and

$$
R=\left[\begin{array}{cc}
\operatorname{diag}\left(\left\|p_{u}-p_{v}\right\|\right) & \mathbf{0} \\
\mathbf{0} & I_{|\mathcal{V}|}
\end{array}\right],
$$

and recall that $\hat{\mathcal{B}}_{\mathcal{G}}(\chi(\mathcal{V}))=R \tilde{\mathcal{B}}_{\mathcal{G}}(\chi(\mathcal{V}))$. Then $Q$ is a $D$ semistable matrix, and in particular, $Q R+R Q \leq 0$ since $R$ is a diagonal and non-negative matrix [21].

The set $\mathcal{S}=\{\delta \mid \dot{V}(\delta)=0\}$ corresponds to points satisfying $b_{\mathcal{G}}(\chi)=\mathbf{b}_{\mathcal{G}}^{d}$ or $\hat{\mathcal{B}}_{\mathcal{G}}(\chi(\mathcal{V}))^{T} \mathbf{b}_{\mathcal{G}}^{d}=0 .{ }^{2}$ The latter occurs only when each entry of $\mathbf{b}_{\mathcal{G}}^{d}$ is in $\operatorname{span}\left\{r_{u v}\right\}$; that is, a scaling of $\left(p^{d}, \psi^{d}\right)$. However, since the scale and centroid are invariant, this set must correspond precisely to the point satisfying $b_{\mathcal{G}}(\chi)=\mathbf{b}_{\mathcal{G}}^{d}$. Therefore, by LaSalle's Invariance Principle, the error dynamics (17) asymptotically converge to the set $\mathcal{S}$. Furthermore, on the set $\mathcal{S}$, the system (16) satisfies $\dot{\chi}(\mathcal{V})=0$.

Some remarks on Theorem III.2 are in order. While Proposition III.1 leads to centroid and scale invariance of the system, we are not able to comment on the final orientation of the formation. Indeed, from Proposition II.7, there should be another invariant of the dynamics that can shed light on the final orientation; we will explore this in future works. Another issue to be concerned with is the possibility of collisions during the system evolution. In fact, it was shown in [16] that for initial conditions sufficiently close to the desired formation, there will be no collisions; we expect this result to hold under the dynamics (16) as well.

\section{SIMULATION RESULTS}

We now report some representative case studies to illustrate the performance of the proposed controller. The first

\footnotetext{
${ }^{2}$ Note that the MIR condition precludes the possibility of any collinear agents.
}

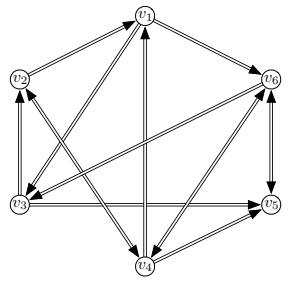

(a)

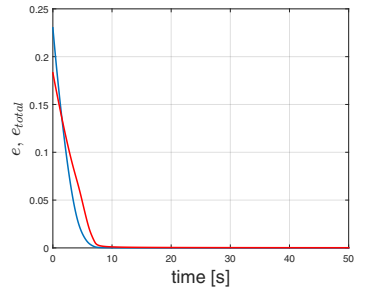

(b)

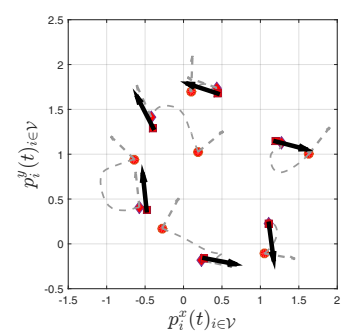

(c)

Fig. 1: The sensing graph in (a) is MIR. (b) Behavior of $e(t)=$ $\frac{1}{|\mathcal{E}|}\left\|b_{\mathcal{G}}(t)-\mathbf{b}_{\mathcal{G}}^{d}\right\|$ (blue solid line) and of $e_{\text {total }}(t)=\frac{1}{|\mathcal{V}|} \| b_{K} K_{|\mathcal{V}|}(t)-$ ${ }_{\mathbf{b}^{d}}^{d}|\mathcal{V}|$ (red solid line). (c) Agent trajectories while converging to the final configuration.

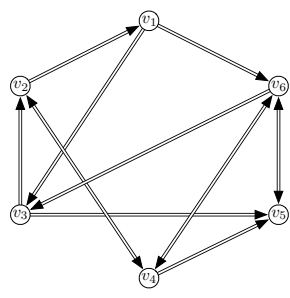

(a)

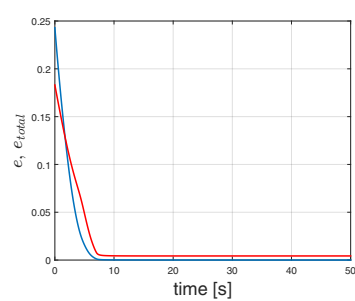

(b)

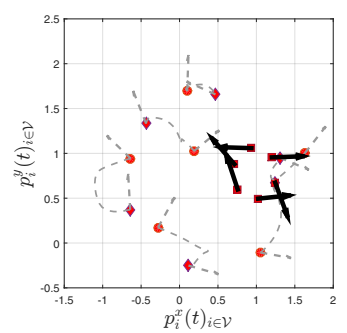

(c)

Fig. 2: The sensing graph in (a) not rigid. The total bearing error $e_{\text {total }}(t)$ does not converge because of the non-rigid framework, and the agents do not reach a configuration congruent with the desired one.

simulation involves $|\mathcal{V}|=6$ agents and the sensing graph $\mathcal{G}$ with $|\mathcal{E}|=14$ shown in Fig. 1(a). This graph is MIR for generic configurations of the agents (in particular, when embedded at the initial condition $\left(p\left(t_{0}\right), \psi\left(t_{0}\right)\right)$ and desired configuration $\left(p^{d}, \psi^{d}\right)$ ). Figure 1(b) reports the superimposed behavior of $e(t)=\frac{1}{|\mathcal{E}|}\left\|b_{\mathcal{G}}(t)-\mathbf{b}_{\mathcal{G}}^{d}\right\|$ (blue solid line) and $e_{\text {total }}(t)=\frac{1}{|\mathcal{V}|}\left\|b_{K_{|\mathcal{V}|}}(t)-\mathbf{b}_{K_{|\mathcal{V}|}}^{d}\right\|$ (red solid line), that is, 


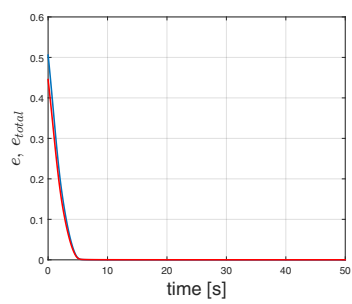

(a)

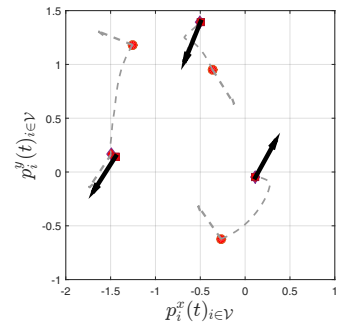

(b)
Fig. 3: A triangular formation with an MIR sensing graph $\mathcal{G}$ with $|\mathcal{E}|=5$ edges. Using Proposition II.10, the relative orientations can be algebraically determined for all agents leading to a pure bearing-only implementation.

the cumulative bearing error over the edge set of graph $\mathcal{G}$ and of the complete graph $K_{|\mathcal{V}|}$, respectively. It is then possible to verify how the bearing controller (12-13) is able to regulate both $e(t)$ and $e_{\text {total }}(t)$ to zero, thus indicating that the 6 agents have successfully reached a configuration congruent with $\mathbf{b}_{\mathcal{G}}^{d}$. Figure $1(\mathrm{c})$ shows the agent trajectories from initial to final configurations. In the plot, dashed gray arrows indicate the initial/final positions/orientations of the agents, while solid black arrows depict a realization of the desired bearing formation rototranslated and scaled so as to match the final pose of agent 1 and the final distance between agents 1 and 2. Again, one can note how all agents correctly approach a final pose consistent with the desired bearing vector $\mathbf{b}_{\mathcal{G}}^{d}$.

Results of the second case study are shown in Figs. 2(ac): here, the sensing graph has $|\mathcal{E}|=13$ edges (one less that in the first case study) and is, thus, not rigid. Consequently, the controller (12-13) is able to zero the bearing error $e(t)$ but not the total bearing error $e_{\text {total }}(t)$ (Fig. 2(b) - note how $e_{\text {total }}(t)$ stabilizes to a constant but non-zero value). Indeed, by looking at Fig. 2(c) one can note how the agents reach a configuration not congruent with the desired one.

Finally, in Figs. 3(a-b), we report the results of a minimally rigid triangular formation with $|\mathcal{V}|=3$ agents and $|\mathcal{E}|=5$ edges in the sensing graph from Proposition II.10. In this case, the control can be implemented without an explicit measurement of the relative orientation using the algebraic characterization described in Proposition II.10.

\section{CONCLUSIONS}

This work examined an extension of the bearing rigidity theory to handle frameworks embedded in $S E(2)$. The structure of the corresponding directed bearing rigidity matrix was examined and turned out to be a central construct in the development of a distributed bearing-only formation control strategy. In particular, we were able to show almost global asymptotic stability of the control strategy when the $S E(2)$ framework is minimally infinitesimally rigid.

\section{ACKNOWLEDGMENTS}

This research was supported by the ANR, Project ANR14-CE27-0007 SenseFly.

\section{REFERENCES}

[1] D. Zelazo, A. Franchi, and P. Robuffo Giordano, "Rigidity Theory in $S E(2)$ for Unscaled Position Estimation using only Bearing Measurements," in European Control Conference, Strasbourg, France, 2014, pp. 2703-2708.

[2] L. Krick, M. E. Broucke, and B. A. Francis, "Stabilisation of infinitesimally rigid formations of multi-robot networks," International Journal of Control, vol. 82, no. 3, p. 423439, 2009.

[3] M.-A. Belabbas, "On global stability of planar formations," IEEE Transactions on Automatic Control, vol. 58, no. 8, 2013.

[4] B. D. O. Anderson, B. Fidan, C. Yu, and D. van der Walle, "UAV formation control: Theory and application," in Recent Advances in Learning and Control, ser. Lecture Notes in Control and Information Sciences, V. D. Blondel, S. P. Boyd, and H. Kimura, Eds. Springer, 2008, vol. 371, pp. 15-34.

[5] K.-K. Oh and H.-S. Ahn, "Formation control of mobile agents based on inter-agent distance dynamics," Automatica, vol. 47, no. 10, pp. $2306-2312,2011$

[6] S. Mou, A. Morse, and B. Anderson, "Toward robust control of minimally rigid undirected formations," in 53rd Conference on Decision and Control, Dec 2014, pp. 643-647.

[7] H. Garcia de Marina, M. Cao, and B. Jayawardhana, "Controlling rigid formations of mobile agents under inconsistent measurements," IEEE Transactions on Robotics, vol. 31, no. 1, pp. 31-39, Feb 2015.

[8] A. N. Bishop, I. Shames, and B. D. Anderson, "Stabilization of rigid formations with direction-only constraints," in IEEE Conference on Decision and Control and European Control Conference, vol. 746, no. 1, Dec. 2011, pp. 746-752.

[9] E. Schoof, A. Chapman, and M. Mesbahi, "Bearing-compass formation control: A human-swarm interaction perspective," in American Control Conference, Portland, USA, June 2014, pp. 3881-3886.

[10] T. Eren, "Formation shape control based on bearing rigidity," Interna tional Journal of Control, vol. 85, no. 9, pp. 1361-1379, Sept. 2012.

[11] A. Franchi and P. Robuffo Giordano, "Decentralized control of parallel rigid formations with direction constraints and bearing measurements," in 51st IEEE Conference on Decision and Control, Dec. 2012, pp. 5310-5317.

[12] A. N. Bishop, I. Shames, and B. D. O. Anderson, "Stabilization of rigid formations with direction-only constraints," in 50th IEEE Conf. on Decision and Control, Orlando, FL, Dec. 2011, pp. 746-752.

[13] T. Eren, W. Whiteley, A. S. Morse, P. N. Belhumeur, and B. D. O. Anderson, "Sensor and network topologies of formations with direction, bearing, and angle information between agents," in 42th IEEE Conf. on Decision and Control, Maui, HI, Dec. 2003, pp. 3064-3069.

[14] P. Gurfil and D. Mishne, "Cyclic spacecraft formations: Relative motion control using line-of-sight measurements only," Journal of Guidance, Control, and Dynamics, vol. 30, no. 1, pp. 214-226, January-February 2007

[15] T. Eren, W. Whiteley, A. S. Morse, P. N. Belhumeur, and B. D. Anderson, "Sensor and Network Topologies of Formations with Direction, Bearing, and Angle Information between Angents," in 42nd IEEE Conference on Decision and Control, 2003, pp. 3064-3069.

[16] S. Zhao and D. Zelazo, "Bearing rigidity and almost global bearingonly formation stabilization," IEEE Transactions on Automatic Control, vol. PP, no. 99, July 2015, (IEEE Early Access Article).

[17] — , "Bearing-Based Distributed Control and Estimation of MultiAgent Systems," in European Control Conference, Linz, Austria, 2015, pp. 2207-2212.

[18] C. D. Godsil and G. Royle, Algebraic Graph Theory. Springer, 2001.

[19] L. Asimow and B. Roth, "The Rigidity of Graphs, II," Journal of Mathematical Analysis and Applications, vol. 68, pp. 171-190, 1979.

[20] A. Franchi, C. Masone, V. Grabe, M. Ryll, H. H. Bülthoff, and P. Robuffo Giordano, "Modeling and control of UAV bearingformations with bilateral high-level steering," The International Journal of Robotics Research, Special Issue on 3D Exploration, Mapping, and Surveillance, vol. 31, no. 12, pp. 1504-1525, 2012.

[21] R. Horn and C. Johnson, Topics in Matrix Analysis. New York, NY: Cambridge University Press, 1991. 